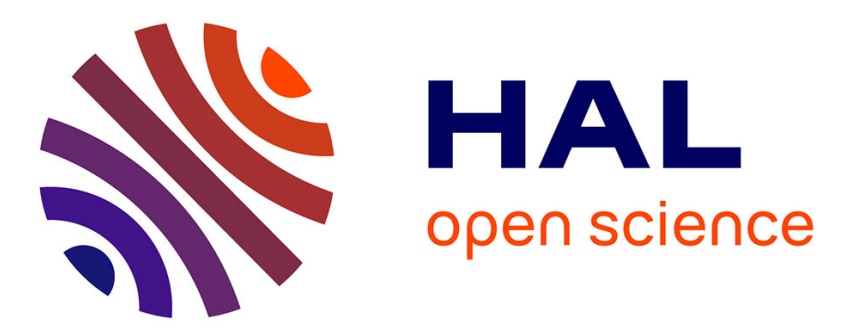

\title{
Non-adiabatic molecular dynamics investigation of the size dependence of the electronic relaxation in polyacenes
}

Evgeny Posenitskiy, Mathias Rapacioli, Bruno Lepetit, Didier Lemoine, Fernand Spiegelman

\section{To cite this version:}

Evgeny Posenitskiy, Mathias Rapacioli, Bruno Lepetit, Didier Lemoine, Fernand Spiegelman. Nonadiabatic molecular dynamics investigation of the size dependence of the electronic relaxation in polyacenes. Physical Chemistry Chemical Physics, 2019, 21 (23), pp.12139-12149. 10.1039/C9CP00603F . hal-02156273v2

\author{
HAL Id: hal-02156273 \\ https://hal.science/hal-02156273v2
}

Submitted on 7 May 2021

HAL is a multi-disciplinary open access archive for the deposit and dissemination of scientific research documents, whether they are published or not. The documents may come from teaching and research institutions in France or abroad, or from public or private research centers.
L'archive ouverte pluridisciplinaire HAL, est destinée au dépôt et à la diffusion de documents scientifiques de niveau recherche, publiés ou non, émanant des établissements d'enseignement et de recherche français ou étrangers, des laboratoires publics ou privés. 


\title{
Non-adiabatic molecular dynamics investigation of the size dependence of the electronic relaxation in polyacenes ${ }^{\dagger}$
}

\author{
Evgeny Posenitskiy, ${ }^{* a}$ Mathias Rapacioli, ${ }^{b}$ Bruno Lepetit, ${ }^{a}$ Didier Lemoine ${ }^{a}$ and Fernand \\ Spiegelman ${ }^{b}$
}

Received Date

Accepted Date

DOI: $10.1039 / x x x x x x x x x x$

www.rsc.org/journalname
The Tully's fewest switches surface hopping algorithm is implemented within the framework of the time-dependent density functional based tight binding method (TD-DFTB) to simulate the energy relaxation following absorption of a UV photon by polycyclic aromatic hydrocarbons (PAHs). This approach is used to study the size effect on the ultrafast dynamics in excited states for a special class of PAH species called polyacenes. We determine the dynamical relaxation times and discuss the underlying mechanisms. Our results show that there is a striking alternation in decay times of the brightest singlet state for neutral polyacenes with 3 to 6 aromatic cycles. The alternation corresponds to an order-of-magnitude variation between roughly 10 and 100 fs and is correlated with a qualitatively similar alternation of energy gaps between the brightest state and the state lying just below in energy.

\section{Introduction}

Modelling the evolution of an extended molecular system following the absorption of a UV photon is a challenging task, which requires a fine description of the energy spreading over many electronic and nuclear degrees of freedom. Understanding the involved processes is however of primary interest to characterize photostability or photochemistry as well as the competition between fluorescence and non-radiative relaxation channels. ${ }^{1}$ The characteristic timescales for such relaxation and the broadening of absorption lines are related because they both rely on the topology of the excited potential energy surfaces (PES).

In the regions where two or more electronic PES are getting close (e.g. in the vicinity of conical intersections), the nuclear and electronic degrees of freedom evolve with similar timescales and cannot be treated separately. Several theoretical approaches exist to cope with such non-adiabatic effects. One consists in

\footnotetext{
${ }^{a}$ Laboratoire Collisions Agrégats et Réactivité (LCAR), IRSAMC UMR5589, Université de Toulouse (UPS) and CNRS, 118 Route de Narbonne, F-31062 Toulouse, France. Email: posenitskiy@irsamc.ups-tlse.fr

${ }^{b}$ Laboratoire de Chimie et Physique Quantiques (LCPQ), IRSAMC UMR5626, Université de Toulouse (UPS) and CNRS, 118 Route de Narbonne, F-31062 Toulouse, France. $\dagger$ Electronic Supplementary Information (ESI) available: Absorption spectra of anthracene, tetracene, pentacene and hexacene computed with TD-DFT and TD-DFTB at the equilibrium geometry (Fig. S1). Energy gaps between the brightest singlet excited state and the one with lower energy in TD-DFTB absorption spectra (Fig. S2). Population analysis for naphthalene with and without the decoherence correction (Fig. S3). Experimental and theoretical data from Fig. 2 (Tab. S1). See DOI: 10.1039/cXCP00000x/
}

propagating the time-dependent wavepacket as done in the multi configuration time-dependent Hartree (MCTDH) scheme. ${ }^{2-4}$ This approach, which makes use of the $a b$ initio PES fitted on a grid, usually allows to take into account only few electronic states and vibrational degrees of freedom due to the computational complexity of the propagation. Alternative schemes have been developed relying on a classical description for nuclei, the two most popular ones being the mean field propagation (Ehrenfest type dynamics ${ }^{5}$ ) and the trajectory surface hopping scheme (e.g. Tully's fewest switches approach ${ }^{6,7}$ ). The latter one requires the calculation of excited electronic states gradients and non-adiabatic couplings, which consumes a significant amount of computational time for large molecules even at the commonly used time-dependent density functional theory (TD-DFT) ${ }^{8}$ level of theory for the electronic structure calculations. The density functional based tight binding (DFTB) ${ }^{9-12}$ is an approximated DFT scheme, whose efficiency relies on the use of parameterized (precomputed) integrals and a small basis set. This method has been extended to access electronic excited states leading to the TD-DFTB approach ${ }^{13}$ - an equivalent of the linear response TDDFT. It has been later on coupled to nuclear dynamics via the trajectory surface hopping scheme to study large molecular systems. ${ }^{14-19}$

The polycyclic aromatic hydrocarbons (PAHs) are relevant molecules in several domains such as combustion, atmospheric and astrophysical sciences. In particular, they have been proposed as the precursors of series of unidentified infrared radiation emission bands ${ }^{20,21}$ and some of the diffuse interstellar absorption 
bands (DIBs), ${ }^{22-24}$ which are ubiquitous in the Universe. The cationic PAHs are considered as better candidates than their neutral counterparts for being DIBs carriers. ${ }^{24-26}$ This motivated several experimental and theoretical investigations of their photoabsorption and dynamical properties. ${ }^{25-35}$ However, less information about the photophysics of neutral PAH molecules is available, despite the fact that they cannot be excluded from contributing to the DIBs. In addition, their photostability has to be studied as well as the efficiency of non-radiative relaxation pathways, which is usually assumed to be fast enough to quickly drive the system into a vibrationally hot electronic ground state (GS). ${ }^{36}$

In the present work, we report a new implementation of the trajectory surface hopping dynamics coupled to the TD-DFTB approach within the deMon-Nano code ${ }^{37}$ and present its first application devoted to PAHs. More precisely, we investigate the dynamical evolution of electronically-excited polyacenes ranging from naphthalene to heptacene, which are systems of choice as they are known to present a closed-shell GS electronic configuration $^{38,39}$ and their absorption spectra have been shown to be well described both at the TD-DFT ${ }^{40}$ and the TD-DFTB ${ }^{13,38}$ levels of theory. The non-adiabatic dynamics of polyacenes following the absorption of a photon in their brightest absorption band is investigated (i) determining the typical timescales of relaxation, (ii) performing analysis in terms of level crossings and evolution with size as well as (iii) estimating the transfer of the electronic energy towards vibrational modes. This work is also motivated by the recent development of experimental tools allowing to probe femtosecond dynamics of this type of molecules. ${ }^{34,35,41-43}$

The paper is organized as follows: in the next section, we briefly outline the basics of DFTB and TD-DFTB as well as some computational and implementation details. Subsequently, the results and discussions are devoted to the application to polyacenes, first assessing the quality of the TD-DFTB for calculating their absorption spectra prior to analyzing the non-adiabatic dynamics for each molecular system. The results are further compiled to emphasize and interpret the size effect. Finally, the conclusions and perspectives are given.

\section{Methods}

The theoretical formulation of the "on-the-fly" non-adiabatic molecular dynamics (NAMD) within the TD-DFTB approach ${ }^{17-19}$ is similar to the one developed for the conventional TD-DFT. ${ }^{44,45}$ In the present section we will give a brief outline of the TD-DFTB formalism and present our implementation of the fewest-switches trajectory surface hopping TD-DFTB in the development version of the deMon-Nano ${ }^{37}$ software package.

\subsection{Linear Response TD-DFTB}

The self-consistent charge DFTB (SCC-DFTB) was developed by Elstner et al. ${ }^{12}$ as an extension of the original DFTB framework. $^{9,10}$ It is based on the 2-nd order expansion of the KohnSham (KS) DFT total energy functional around a reference electronic density $n_{0}$, so the final expression for the DFTB total energy reads

$$
E_{\mathrm{SCC}}=\sum_{i=1}^{N_{o c c}} n_{i} \sum_{\mu \nu} a_{\mu i} H_{\mu \nu}^{0} a_{v i}+\frac{1}{2} \sum_{A=1}^{N_{a t}} \sum_{B=1}^{N_{a t}} \Delta q_{A} \gamma_{A B} \Delta q_{B}+E_{r e p},
$$

where $n_{i}$ is the occupation number of molecular orbital (MO) $i, \mu$ and $v$ are the KS atomic orbital (AO) indices, $\Delta q_{A}$ is the Mulliken charge of atom A, $E_{\text {rep }}$ is the atomic pair repulsive contribution, $a_{\mu i}$ are KS MO coefficients and $\gamma_{A B}$ describes the Coulomb interaction between spherically symmetric charge distributions centered on the atoms $\mathrm{A}$ and $\mathrm{B}$ with a short range exchangecorrelation contribution. The total energy $E_{\mathrm{SCC}}$ is further minimized following the self-consistent procedure as proposed by Elstner et al. ${ }^{12}$

The main success of the DFTB method was due to a significant reduction of the computational time for an electronic structure calculation, which was achieved by precomputing all necessary matrix elements for different interatomic distances based on DFT calculations. More detailed introduction to DFTB can be found in refs. 46, 47.

Linear response TD-DFTB was developed by Niehaus et al. ${ }^{13}$ as a DFTB analogue of the conventional linear response TD-DFT. ${ }^{8,48}$ Excitation energies are given as eigenvalues $\Omega_{I}$ of the following matrix equation:

$$
\left(\begin{array}{ll}
\mathbf{A} & \mathbf{B} \\
\mathbf{B} & \mathbf{A}
\end{array}\right)\left(\begin{array}{l}
\mathbf{X} \\
\mathbf{Y}
\end{array}\right)=\Omega_{I}\left(\begin{array}{cc}
\mathbb{1} & 0 \\
0 & -\mathbb{1}
\end{array}\right)\left(\begin{array}{l}
\mathbf{X} \\
\mathbf{Y}
\end{array}\right),
$$

where $\mathbb{1}$ is the identity matrix, $\mathbf{A}$ and $\mathbf{B}$ are matrices with the elements given by

$$
\begin{gathered}
A_{i a, j b}=\left(\varepsilon_{a}-\varepsilon_{i}\right) \delta_{i j} \delta_{a b}+2 K_{i a, j b} ; \\
B_{i a, j b}=2 K_{i a, j b} ;
\end{gathered}
$$

and indices $i, j$ and $a, b$ denoting the occupied and virtual MOs with energies $\varepsilon_{i}$ and $\varepsilon_{a}$, respectively. The coupling matrix elements $K_{i a, j b}$ are calculated within the DFTB approach using the generalized Mulliken approximation. ${ }^{13}$

\subsection{Fewest-switches trajectory surface hopping (FSSH)}

Standard molecular dynamics usually relies on the BornOppenheimer approximation, which retains a single adiabatic electronic state in the expansion of the wavefunction of the system. Several approaches have been developed in recent years to incorporate the non-adiabatic effects both at the ab-initio ${ }^{49}$ and mixed quantum-classical ${ }^{50-53}$ levels of theory. In this article, we use Tully's FSSH scheme. ${ }^{6,7}$ It is a quantum-classical approach to quantum dynamics, with the nuclear wavepacket motion simulated by an ensemble of independent classical trajectories, each one evolving on a single electronic state at a given time. The principles of the present implementation are briefly summarized hereafter.

In the Tully's scheme, the electronic wavefunction $\Psi$ is expanded on a basis of $N_{s t}$ adiabatic electronic states

$$
\Psi(\mathbf{r} ; \mathbf{R}(t))=\sum_{J=1}^{N_{s t}} C_{J}(t) \psi_{J}(\mathbf{r} ; \mathbf{R}(t)),
$$


where $C_{J}$ is the complex expansion coefficient and $\psi_{J}(\mathbf{r} ; \mathbf{R}(t))$ is the adiabatic electronic wavefunction of state $J$, which depends on the electronic coordinates $\mathbf{r}$ and parametrically on the nuclear coordinates $\mathbf{R}(t)$. We denote $\psi_{J}(\mathbf{r} ; \mathbf{R}(t))=\psi_{J}(t)$ for simplicity.

Substituting eqn (5) into the time-dependent electronic Schrödinger equation and taking into account the adiabatic nature of states, one derives the following equation for the propagation of the expansion coefficients $C_{J}(t)$ :

$$
i \hbar \frac{\mathrm{d} C_{J}(t)}{\mathrm{d} t}=C_{J}(t) E_{J}(t)-i \hbar \sum_{K \neq J} C_{K}(t) D_{J K}(t),
$$

where $E_{J}$ is the adiabatic energy of state $J, D_{J K}=\left\langle\psi_{J} \mid \frac{\partial \psi_{K}}{\partial t}\right\rangle$ is the non-adiabatic coupling (NAC) between states $J$ and $K$. In our implementation the NAC is calculated using a finite difference method: ${ }^{7}$

$$
D_{J K}(t+\Delta t / 2) \approx \frac{1}{2 \Delta t}\left[\left\langle\psi_{J}(t) \mid \psi_{K}(t+\Delta t)\right\rangle-\left\langle\psi_{J}(t+\Delta t) \mid \psi_{K}(t)\right\rangle\right] .
$$

It is important to apply the decoherence correction on $C_{J}$ since the propagation of eqn (6) in FSSH is overcoherent, which means that electronic coherence $C_{I} C_{J}^{*}$ do not vanish after passing through the region of strong NAC between states $I$ and $J$. Decoherence corrections have been shown to be crucial in a number of applications. ${ }^{54,55}$ We use the simplified decay of mixing method ${ }^{56}$ to correct the $C_{J}$ coefficients:

$$
\begin{gathered}
\widetilde{C}_{J}=C_{J} \mathrm{e}^{-\Delta t / \tau_{I I}} \quad \forall J \neq I ; \\
\widetilde{C}_{I}=\frac{C_{I}}{\left|C_{I}\right|} \sqrt{1-\sum_{J \neq I}\left|\widetilde{C}_{J}\right|^{2}},
\end{gathered}
$$

where $I$ denotes the active state, namely the one on which the trajectory is evolving at the considered time, and $\tau_{J I}$ is the decoherence time, which can be determined from the following equation:

$$
\tau_{J I}=\frac{\hbar}{\left|\Delta E_{J I}\right|}\left(1+\frac{\alpha}{E_{k i n}}\right)
$$

where $E_{k i n}$ is nuclear kinetic energy, $\Delta E_{J I}=E_{J}-E_{I}$ and $\alpha$ is a phenomenological parameter (recommended value ${ }^{56}$ is 0.1 hartree).

Once both electrons and nuclei are propagated from $t$ to $t+\Delta t$ and decoherence corrections applied, the probability to switch from the active state $I$ to another state $K$ during electronic time step $\Delta \tau$ is estimated from the following equation: ${ }^{19}$

$$
P_{I \rightarrow K}(\tau)=\max \left[0 ;-2 \Delta \tau \frac{\operatorname{Re}\left(\widetilde{C}_{I}^{*}(\tau) \widetilde{C}_{K}(\tau)\right)}{p_{I}} D_{I K}(\tau)\right]
$$

where $p_{I}=\left|\widetilde{C}_{I}\right|^{2}$ is the population of a given state $I . P_{I \rightarrow K}(\tau)$ computed for $t \leqslant \tau \leqslant t+\Delta t$ determines the final hopping probability via an integration over $\tau: 44,45$

$$
\widetilde{P}_{I \rightarrow K}(t+\Delta t) \approx \int_{t}^{t+\Delta t} \frac{P_{I \rightarrow K}(\tau)}{\Delta \tau} d \tau .
$$

In our implementation, hops may only occur at the nuclear time scale for it is consistent with the mixed quantum-classical formulation of the problem. A uniform random number $0<\xi<1$ is generated at each nuclear time step to determine if the hop from the current state $I$ to any state $K$ is allowed from the quantum point of view. ${ }^{7}$ The hop is accepted if the following condition is fulfilled:

$$
\begin{gathered}
\sum_{J=1}^{K-1} \widetilde{P}_{I \rightarrow J}<\xi \leqslant \sum_{J=1}^{K} \widetilde{P}_{I \rightarrow J} \quad K \neq 1 ; \\
\xi \leqslant \widetilde{P}_{I \rightarrow 1} \quad \text { otherwise. }
\end{gathered}
$$

To conserve the total energy after hopping, the nuclear velocities are rescaled uniformly by a factor $\beta$ following the energy conservation law:

$$
E_{I}+E_{k i n}=E_{J}+\beta^{2} E_{k i n} .
$$

Thus, the switch of two states can be still rejected if the energy gap $E_{J}-E_{I}>E_{k i n}$. Such hops are called "frustrated" or classically forbidden hops.

\subsection{Dynamical quantities within TD-DFTB}

In order to propagate trajectory on a given PES, the excited states energy gradients have to be developed for the linear response TDDFTB. Elstner et al. introduced analytical GS forces at the SCCDFTB level of theory. ${ }^{12}$ Further efforts were made to extract analytical excitation energy gradients from eqn (2). Its derivation relies on the so-called Z-vector method, which was initially applied by Furche and Ahlrichs ${ }^{57,58}$ to compute analytical excited states forces within the TD-DFT approach. This procedure was further used to derive TD-DFTB gradients by Heringer et al. ${ }^{59,60}$ and led to the final expression published in ref. 38. We recommend to follow the procedure introduced in refs. 59, 60 to calculate all necessary matrix elements in the MO representation and then use eqn (15) from ref. 38 to compute the excitation energy gradients in the AO representation, which is more consistent with the DFTB formalism.

NAC is one of the key quantities of FSSH for it determines the propagation of the electronic populations and hopping probabilities. DFTB, as a density functional method, was not initially developed for the wavepacket calculations. Thus, it is not clear how to build electronic wavefunctions of excited states, which are used in eqn (7). Common practice is to use the configuration interaction singlets (CIS) approach to derive the electronic wavefunction of state $K: 16,19,44,45,48,61,62$

$$
\left|\psi_{K}\right\rangle=\sum_{i \rightarrow a}^{N_{t r}} c_{i a}^{K}\left|\Phi_{i a}^{0}\right\rangle,
$$

where $c_{i a}^{K}$ is CIS expansion coefficient and $\left|\Phi_{i a}^{0}\right\rangle$ is a singly excited Slater determinant (SD) built from a given electronic transition $i \rightarrow a$. We further followed the procedure, which is described 
in great details by Humeniuk and Mitrić (see Appendix C in ref. 16), to extract the electronic wavefunctions overlap between $t$ and $t+\Delta t$ based on the SD overlap:

$$
\left\langle\psi_{J}(t) \mid \psi_{K}(t+\Delta t)\right\rangle=\sum_{i \rightarrow a}^{N_{t r}} \sum_{j \rightarrow b}^{N_{t r}} c_{j b}^{J}(t) c_{i a}^{K}(t+\Delta t)\left\langle\Phi_{j b}^{0}(t) \mid \Phi_{i a}^{0}(t+\Delta t)\right\rangle .
$$

We also implemented two different ways to compute $c_{i a}^{K}$, which are commonly used in the literature:

1. $c_{i a}^{K}=(X+Y)_{i a}^{K}=\sqrt{\frac{\varepsilon_{a}-\varepsilon_{i}}{\Omega_{K}}} F_{i a}^{K}$ - was used by Tapavicza et al. ${ }^{44}$ following Casida's assignment from ref. 48. Excited states are not orthogonal in that case;

2. $c_{i a}^{K}=F_{i a}^{K}$ - was proposed by Werner et al. ${ }^{62}$ Excited states are orthogonal,

where $F_{i a}^{K}$ is the eigenvector of eqn (2) for the transition $i \rightarrow a$ within the given state $K$. In our calculations, the second set is used for it provides orthogonal excited states. It is worth mentioning, that certain choices of CIS coefficients may lead to the overor underestimation of NACs and hopping probabilities. However, comparison of different techniques used to compute NACs and their impact on NAMD are beyond the scope of this paper.

\subsection{Computational details}

Eqn (6) is integrated using a 4-th order Runge-Kutta algorithm with an electronic time step $\Delta \tau=0.048$ as. Each classical trajectory is propagated with $\Delta t=0.25 \mathrm{fs}$ during $300 \mathrm{fs}$. Adiabatic energies and NACs are only available at nuclear time steps $t, t+\Delta t$ and $t+\Delta t / 2$, respectively. So in order to integrate eqn (6) with the smaller time step $\Delta \tau$ we interpolate the energies in the time inter$\operatorname{val}(t, t+\Delta t)$ and suppose $D_{J K}(\tau)=D_{J K}(t+\Delta t / 2)$ for $t \leqslant \tau \leqslant t+\Delta t$.

Energies are computed only along the trajectory, thus our PES is a $1 \mathrm{D}$ cross-section of a multidimensional one. FSSH cannot deal with the conical intersections (CoIn) due to the singularity of NAC at the point of CoIn. However, several techniques were developed to cope with it. The most straightforward way is to switch states once the energy gap between them drops below a given threshold, thus avoiding numerical instabilities, which occur due to the singularity of NAC. In our simulations, we use a threshold of $0.01 \mathrm{eV}$ for CoIn between excited singlet states and $0.1 \mathrm{eV}$ to detect CoIn between excited singlet and ground states. The latter value was taken larger due to a well-known issue of the inappropriate $S_{1} / S_{0}$ PES topology in TD-DFT. ${ }^{45}$ One can also terminate the trajectory once CoIn with GS occurs. We would like to emphasize that no CoIn with GS occurred during our NAMD simulations for polyacenes. Another alternative is to use the local diabatization technique. ${ }^{63}$

Initial conditions were sampled from the thermal distribution of the GS. Single trajectory was equilibrated at $T=300 \mathrm{~K}$ during 50 ps using a chain of 5 Nosé-Hoover thermostats and $0.5 \mathrm{fs}$ time step. Snapshots were taken every 50 fs to be further used as initial conditions for the NAMD. The DFTB parameters were taken from the mio-set. ${ }^{12}$ As an example, 63 initial configurations sampled for tetracene at $T=300 \mathrm{~K}$ are presented in Fig. 1. For a more detailed discussion on the choice of initial conditions sampling in the excited states dynamics see refs. 53, 64.

We use a threshold to truncate the number of transitions involved in the CIS expansion. Our benchmark calculations demonstrate some numerical instabilities if we exclude all $i \rightarrow a$ transitions with $\left|c_{i a}^{K}\right|<10^{-2}$ and provide reasonably accurate results already for $\left|c_{i a}^{K}\right|<10^{-3}$. This truncation may reduce a number of transitions in eqn (16) by an order of magnitude and significantly accelerate the computation of NACs for large molecules.

Furthermore, we would like to clarify some computational details, which are related to the NAMD itself. An ensemble of 63 trajectories was used for all calculations (except naphthalene and anthracene) presented in Section 3. Our benchmark simulations for naphthalene and anthracene with 127 and 63 trajectories demonstrate that the latter is large enough to reproduce primary relaxation channels in polyacenes. The maximum statistical error depends on the amount of trajectories $N_{\text {traj }}$ and for $95 \%$ confidence interval reads 53

$$
\varepsilon \approx \frac{0.98}{\sqrt{N_{t r a j}}}
$$

Thus, we are not able to resolve any process, which occurs with a probability lower than $12 \%$.

\section{Results and discussion}

\subsection{Absorption spectra of polyacenes}

All polyacenes have the $D_{2 h}$ point group symmetry and a GS belonging to the $A_{g}$ irreducible representation, thus their brightest excited states for an electric dipolar transition should belong to the $B_{3 u}$ irreducible representation if we align the considered molecules along $x$ axis.

It was previously shown that the TD-DFTB is able to represent key features of the absorption spectra of polyacenes. ${ }^{13}$ However, we have repeated these calculations first with a different DFT functional and second with a different set of DFTB parameters, mainly to evaluate an accuracy of the latter for we will use it in the present NAMD calculations. The TD-DFT absorption spectra were computed with Gaussian 09 package ${ }^{65}$ using BLYP functional and 6-31G(d,p) basis set while the TD-DFTB data were calculated with the deMon-Nano ${ }^{37}$ code using matsci-0-3 ${ }^{66}$ set of parameters. All geometries used in the TD-DFT were optimized with the B3LYP functional and $6-311 \mathrm{G}^{*}$ basis set, TD-DFTB geometries were optimized using matsci-0-3.

We further validate our theoretical data based on comparison with experimental values. All experimental absorption spectra data for naphthalene, anthracene, tetracene and pentacene are taken from ref. 67 and references therein, for hexacene and heptacene from ref. 68, for octacene from ref. 69.

It is clear from Fig. 2 that the brightest and the first singlet excited states in TD-DFTB and TD-DFT are both in good agreement with experimental values. However, some departure grows with increasing size of the molecule: for octacene the difference is 0.53 $\mathrm{eV}$ and $0.39 \mathrm{eV}$ for the first and brightest singlet excited states, respectively. Thus, we limit ourselves to the study of heptacene 
( $n=7)$ since the brightest singlet excited state is still predicted reasonably well and underestimates the experimental value by $0.22 \mathrm{eV}$ only. More details about the absorption spectra of some polyacenes computed with TD-DFT and TD-DFTB can be found in the ESI. $\dagger$

\subsection{NAMD of polyacenes}

We distinguish between polyacenes with odd and even number of aromatic cycles based on their dynamical behaviour during the relaxation.

\subsubsection{Odd number of aromatic cycles.}

In this section, we present the results of the TD-DFTB FSSH calculations for polyacenes with an odd number of aromatic cycles. The brightest singlet excited states in the TD-DFTB absorption spectra of anthracene, pentacene and heptacene are $S_{7}, S_{10}$ and $S_{11}$, respectively. We start with the analysis of NAMD in anthracene computed with ensembles of different sizes.

We conclude from Fig. 3 that 63 trajectories are enough to fit the decay of the initial state and that the increase in the number of trajectories mainly results in smoothing of the population curves. The general trends of the population transfer (e.g. fast decay of an initial state and the corresponding transfer to lowest singlets) are already represented with 63 trajectories. The decay times extracted from the exponential fits are close to $22 \mathrm{fs}$ in both cases.

The population transfer in pentacene is very similar to the one in anthracene, showing ultrafast decay (within approximately 8 fs) of the initial state and a rapid growth of the population in the lower-lying singlets. However, in heptacene, the latter trend is less pronounced due to a significant transfer of population to $S_{12}$ (see the purple curve during first $50 \mathrm{fs}$ ). Thus, a part of the population is trapped for some time in the upper states resulting in a delay of the initial state decay. Another difference is that more than $65 \%$ of the population spreads over the lower-lying states after 300 fs for both anthracene and pentacene, in comparison with about $55 \%$ for heptacene.

\subsubsection{Even number of aromatic cycles.}

In this section, we present results of the TD-DFTB FSSH calculations for polyacenes with an even number of aromatic cycles. The brightest singlet excited states in the TD-DFTB absorption spectra of naphthalene, tetracene and hexacene are $S_{4}, S_{7}$ and $S_{10}$, respectively.

The electronic population in tetracene is mainly shared between $S_{5}, S_{6}, S_{7}$ and $S_{8}$ during the first 70 fs and then $S_{5}$ (orange line in Fig. 5b) is monotonously populated until it reaches approximately $40 \%$ of population. In hexacene, the main relaxation channel goes through the lower-lying singlet states (blue line in Fig. 5c) after $50 \mathrm{fs}$, while population of each of the neighbouring states $\left(S_{8}, S_{9}, S_{11}\right)$ amounts to about $10 \%$. Nevertheless, both tetracene and hexacene demonstrate slow decay ( 96 and 89 fs, respectively) of the initial singlet excited state for which the population drops below $20 \%$ after 200 fs of propagation.

Analysis for naphthalene is slightly more complicated since the populations of all excited states involved in the propagation reach a plateau around 20\%. The main difference is that after $200 \mathrm{fs} S_{1}$ and $S_{2}$ start sharing approximately $50 \%$ of population. Thus, we conclude that naphthalene has the highest energy transfer rate among all considered polyacenes. This can be also seen if we plot the decrease of the electronic potential energy versus the corresponding increase of the total nuclear kinetic energy along the trajectory. From Fig. 6 we conclude that $0.6 \mathrm{eV}$ on average is transferred from electrons to nuclei in naphthalene after $300 \mathrm{fs}$ of propagation, while in anthracene only $0.25 \mathrm{eV}$ is deposited into the nuclear degrees of freedom.

\subsection{Discussion}

Fig. 7 displays the decay time of the brightest singlet excited state as a function of number of aromatic cycles. All values were extracted from Figs. 3-5.

Decay times can be extracted from exponential fits of the population curves computed with initial conditions starting from the brightest singlet excited state, namely that with the largest oscillator strength (see Fig. S1 in the ESI $\dagger$ ). Although the decay is not fully exponential, our approximate fits on the full time window allow for quantitative comparison between polyacenes. We would like to emphasize that decay times plotted in Fig. 7 are within the same order of magnitude (except for naphthalene) as the ones reported for cationic species by Reddy et al. in ref. 31 even though cationic states crossings could be qualitatively different from those in the neutral molecules. However, the present results exhibit an unexpected and striking alternation of the decay times for an even-odd number of cycles in the range of $n=3-6$. From the organic photochemistry perspective, ${ }^{1}$ one could expect a monotonous decrease of the relaxation time as a function of size governed by the energy gap configurations and an increasing density of states. In order to explain this alternation, we computed the energy gap between the initial state and the neighbour state with lower energy for polyacenes with $n=3-6$ aromatic cycles. It provides a good understanding of how the initial state population transfer occurs due to the fact that during relaxation most of the trajectories either undergo surface hopping in the region of avoided crossing with the state below or reach the CoIn with this state.

It is clear from Fig. 8 that during the first 20 fs a large fraction of trajectories in anthracene and pentacene either go to the region of small gaps $(<0.05 \mathrm{eV})$ or through the CoIn. Thus, there is a funnel in both polyacenes, which drives the rapid population transfer from the initial state. On the contrary, in tetracene, very few trajectories reach the region of small gaps over $20 \mathrm{fs}$ and the averaged value is almost twice larger than the one in anthracene. The latter is also true for hexacene, for which there is a clear drop of the energy gap around $10 \mathrm{fs}$, yet remaining larger than in pentacene.

We have also performed TD-DFTB calculations of the absorption spectra at the equilibrium geometry for nonacene and decacene (see Fig. S2 in the ESI $\dagger$ ) to see whether the energy gap alternation between the brightest and the next lower singlet excited states still occurs for larger polyacenes. The decay alternation cannot be explained with a simple single particle excitation model. This can be seen from the red curve in Fig. S2 (see ESI $\dagger$ ), 
which represents the energy gap as a function of size before the TD-DFTB coupling matrix splits degenerate excitations from occupied to virtual orbitals. The general trend is a monotonous (except for anthracene and octacene) growth with increasing number of aromatic cycles. However, the energy gaps after splitting (blue curve in Fig. S2, see ESI $\dagger$ ) significantly differ between heptacene and octacene. Thus, one can expect a faster decay of the brightest singlet excited states for $n=8,9,10$ when compared to $n=6,7$.

Our results for polyacenes violate Kasha's rule for the nonradiative decay to the $S_{1}$ state. $^{70}$ There are several possible reasons for this: (i) for isolated molecules the lack of dissipative modes to the environment results in the possibility of population back-transfer to higher-lying excited states from the underlying ones; ${ }^{19}$ (ii) 300 fs is a rather short timescale for NAMD, especially for the relaxation from higher-lying excited states, and some trajectories remain in the states above $S_{1}$ at the end of the simulation; (iii) conjugated systems like polyacenes have some lowlying excited states that are dominated by double excitations, ${ }^{71}$ which are missing in the linear response TD-DFTB. Decoherence correction could be thought of as another reason behind the nonvanishing populations above $S_{1}$. We repeated the calculations for naphthalene but without the decoherence correction (see Fig. S3 in the ESI $\dagger$ ). Not only the trend remains the same but furthermore the $S_{1}$ population that ends as the largest state population in Fig. 5, remains the smallest one without correction, thereby enhancing the violation of Kasha's rule. Last, it is worth mentioning that experimental evidence of frequent non-Kasha behaviors is well documented, in particular for small size polyatomic molecules including naphthalene. ${ }^{72}$

Intersystem crossings (ISC) and the corresponding dynamics in excited triplet states have not been studied in the present work. The efficiency of ISC in pure aromatic compounds has been debated in the literature. ${ }^{73,74}$ Spin-orbit coupling is relatively small in molecules involving light atoms only. Yet, it has been shown that ISC could be significantly enhanced, not only in the presence of heavy atoms, ${ }^{75}$ but also upon departure from planarity in aromatic compounds associated with radicals. ${ }^{76}$ Addressing ISC would require the coupling of both triplet and singlet TDDFTB states in the dynamics via the spin-orbit coupling, which is not yet implemented in our code and would be computationally much more demanding. Last, the ISC timescale is most likely to be larger than our simulation timescale by several orders of magnitude.

\section{Conclusion}

In this article, a detailed theoretical study of the non-adiabatic molecular dynamics launched in the brightest singlet excited state has been presented for a set of polyacenes. The results have been obtained using Tully's FSSH scheme coupled to the TD-DFTB formalism for electronic structure calculations.

Relaxation from the brightest singlet excited states via the cascade of radiationless transitions is reported for polyacenes ranging in size from naphthalene to heptacene. The detailed analysis reveals a striking (by an order of magnitude) alternation of decay times computed for linear PAH species with $n=3-6$ aro- matic cycles. Indeed, the calculated values for anthracene and pentacene are about 10-20 fs whereas those for tetracene and hexacene are about $90-100$ fs. This alternation is correlated with the energy gap between the initial state and the neighbouring one with lower energy. Yet, an even-odd alternation in the population transfer cannot be extrapolated to larger systems, even though this effect is well pronounced for polyacenes with $n=3-6$ aromatic cycles. Further investigation is possible, but requires some improvements in the DFTB parametrization to better match the absorption spectra of octacene and larger species. Also, the computational cost of NAMD calculations for larger polyacenes will increase significantly due to a rapidly increasing amount of states to be taken into account.

This research contributes to the understanding of photophysics of PAHs and, more particularly, sheds light on the photostability of considered polyacenes following the absorption of a UV photon from young stars. As far as we know, this study is the first to investigate the relaxation from the higher-lying singlet states of large neutral PAH species like heptacene.

\section{Conflicts of interest}

There are no conflicts to declare.

\section{Acknowledgements}

We acknowledge the European Union (EU) and Horizon 2020 funding awarded under the Marie Skłodowska-Curie action to the EUROPAH consortium, grant number 722346. This work was performed using HPC resources from CALMIP (Grant 2018-P18019). E.P. is grateful to Professor Thomas Niehaus and Aude Simon for fruitful discussions.

\section{References}

1 M. Klessinger and J. Michl, Excited States and Photo-Chemistry of Organic Molecules, Wiley, 1995.

2 H.-D. Meyer, U. Manthe and L. Cederbaum, Chemical Physics Letters, 1990, 165, 73-78.

3 M. Beck, A. Jäckle, G. Worth and H.-D. Meyer, Physics Reports, 2000, 324, 1-105.

4 D. Mendive-Tapia, B. Lasorne, G. A. Worth, M. A. Robb and M. J. Bearpark, The Journal of Chemical Physics, 2012, 137, 22A548.

5 P. Ehrenfest, Zeitschrift für Physik, 1927, 45, 455-457.

6 J. C. Tully, The Journal of Chemical Physics, 1990, 93, 10611071.

7 S. Hammes-Schiffer and J. C. Tully, The Journal of Chemical Physics, 1994, 101, 4657-4667.

8 M. E. Casida, Journal of Molecular Structure: THEOCHEM, 2009, 914, 3-18.

9 D. Porezag, T. Frauenheim, T. Köhler, G. Seifert and R. Kaschner, Phys. Rev. B, 1995, 51, 12947-12957.

10 G. Seifert, D. Porezag and T. Frauenheim, Int. J. Quantum Chem., 1996, 58, 185-192.

11 M. Elstner and G. Seifert, Phil. Trans. R. Soc. A, 2014, 372, 20120483.

12 M. Elstner, D. Porezag, G. Jungnickel, J. Elsner, M. Haugk, 
T. Frauenheim, S. Suhai and G. Seifert, Phys. Rev. B, 1998, 58, 7260-7268.

13 T. A. Niehaus, S. Suhai, F. Della Sala, P. Lugli, M. Elstner, G. Seifert and T. Frauenheim, Phys. Rev. B, 2001, 63, 085108.

14 E. Titov, A. Humeniuk and R. Mitrić, Phys. Chem. Chem. Phys., 2018, 20, 25995-26007.

15 J. Hoche, H.-C. Schmitt, A. Humeniuk, I. Fischer, R. Mitrić and M. I. S. Röhr, Phys. Chem. Chem. Phys., 2017, 19, 2500225015.

16 A. Humeniuk and R. Mitrić, Computer Physics Communications, 2017, 221, 174-202.

17 R. Mitrić, U. Werner, M. Wohlgemuth, G. Seifert and V. Bonačić-Koutecký, The Journal of Physical Chemistry A, 2009, 113, 12700-12705.

18 S. Pal, D. J. Trivedi, A. V. Akimov, B. Aradi, T. Frauenheim and O. V. Prezhdo, Journal of Chemical Theory and Computation, 2016, 12, 1436-1448.

19 L. Stojanović, S. G. Aziz, R. H. Hilal, F. Plasser, T. A. Niehaus and M. Barbatti, Journal of Chemical Theory and Computation, 2017, 13, 5846-5860.

20 A. Léger and J. L. Puget, Astron. Astrophys., 1984, 137, L5-L8.

21 L. J. Allamandola, A. G. G. M. Tielens and J. R. Barker, Astrophys. J., 1985, 290, L25-L28.

22 G. P. van der Zwet and L. J. Allamandola, Astron. Astrophys., 1985, 146, 76-80.

23 A. Léger and L. D’Hendecourt, Astron. Astrophys., 1985, 146, 81-85.

24 M. K. Crawford, A. G. G. M. Tielens and L. J. Allamandola, Astrophys. J. Lett., 1985, 293, L45-L48.

25 F. Salama, G. A. Galazutdinov, J. Krełowski, L. J. Allamandola and F. A. Musaev, Astrophys. J., 1999, 526, 265-273.

26 F. Salama, E. L. O. Bakes, L. J. Allamandola and A. G. G. M. Tielens, Astrophys. J., 1996, 458, 621.

27 K. F. Hall, M. Boggio-Pasqua, M. J. Bearpark and M. A. Robb, The Journal of Physical Chemistry A, 2006, 110, 1359113599.

28 A. M. Tokmachev, M. Boggio-Pasqua, M. J. Bearpark and M. A. Robb, The Journal of Physical Chemistry A, 2008, 112, 1088110886.

29 A. M. Tokmachev, M. Boggio-Pasqua, D. Mendive-Tapia, M. J. Bearpark and M. A. Robb, The Journal of Chemical Physics, 2010, 132, 044306.

30 V. S. Reddy, S. Ghanta and S. Mahapatra, Physical Review Letters, 2010, 104, 111102-.

31 S. N. Reddy and S. Mahapatra, The Journal of Physical Chemistry A, 2013, 117, 8737-8749.

32 S. Ghanta, V. S. Reddy and S. Mahapatra, Phys. Chem. Chem. Phys., 2011, 13, 14523-14530.

33 S. Ghanta, V. S. Reddy and S. Mahapatra, Phys. Chem. Chem. Phys., 2011, 13, 14531-14541.

34 A. Marciniak, V. Despré, T. Barillot, A. Rouzée, M. C. E. Galbraith, J. Klei, C. H. Yang, C. T. L. Smeenk, V. Loriot, S. N. Reddy, A. G. G. M. Tielens, S. Mahapatra, A. I. Kuleff, M. J. J. Vrakking and F. Lépine, Nature Communications, 2015, 6,
7909.

35 A. Marciniak, V. Despré, V. Loriot, G. Karras, M. Hervé, L. Quintard, F. Catoire, C. Joblin, E. Constant, A. I. Kuleff and F. Lépine, Nature Communications, 2019, 10, 337.

36 A. Léger, L. D’Hendecourt and D. Defourneau, Astron. Astrophys., 1989, 216, 148-164.

37 T. Heine, M. Rapacioli, S. Patchkovskii, J. Frenzel, A. M. Köster, P. Calaminici, H. A. Duarte, S. Escalante, R. FloresMoreno, A. Goursot, J. U. Reveles, D. R. Salahub and A. Vela, deMonNano, 2009, http://demon-nano.ups-tlse.fr/.

38 R. Rüger, T. Niehaus, E. van Lenthe, T. Heine and L. Visscher, The Journal of Chemical Physics, 2016, 145, 184102.

39 Y. Yang, E. R. Davidson and W. Yang, Proceedings of the National Academy of Sciences, 2016, 113, E5098-E5107.

40 M. L. M. Rocco, M. Häming, C. E. V. de Moura, M. Barbatti, A. B. Rocha, A. Schöll and E. Umbach, The Journal of Physical Chemistry C, 2018, 122, 28692-28701.

41 M. C. E. Galbraith, S. Scheit, N. V. Golubev, G. Reitsma, N. Zhavoronkov, V. Despré, F. Lépine, A. I. Kuleff, M. J. J. Vrakking, O. Kornilov, H. Köppel and J. Mikosch, Nature Communications, 2017, 8, 1018.

42 M. C. E. Galbraith, C. T. L. Smeenk, G. Reitsma, A. Marciniak, V. Despré, J. Mikosch, N. Zhavoronkov, M. J. J. Vrakking, O. Kornilov and F. Lépine, Phys. Chem. Chem. Phys., 2017, 19, 19822-19828.

43 V. Loriot, A. Marciniak, L. Quintard, V. Despré, B. Schindler, I. Compagnon, B. Concina, G. Celep, C. Bordas, F. Catoire, E. Constant and F. Lépine, Journal of Physics: Conference Series, 2015, 635, 012006.

44 E. Tapavicza, I. Tavernelli and U. Rothlisberger, Phys. Rev. Lett., 2007, 98, 023001.

45 E. Tapavicza, I. Tavernelli, U. Rothlisberger, C. Filippi and M. E. Casida, The Journal of Chemical Physics, 2008, 129, 124108.

46 A. F. Oliveira, G. Seifert, T. Heine and H. A. A. Duarte, Journal of the Brazilian Chemical Society, 2009, 20, 1193-1205.

47 P. Koskinen and V. Mäkinen, Computational Materials Science, 2009, 47, 237-253.

48 M. E. Casida, in Time-Dependent Density Functional Response Theory for Molecules, 1995, pp. 155-192.

49 B. F. E. Curchod and T. J. Martínez, Chemical Reviews, 2018, 118, 3305-3336.

50 E. Fabiano, T. Keal and W. Thiel, Chemical Physics, 2008, 349, 334-347.

51 M. Persico and G. Granucci, Theoretical Chemistry Accounts, 2014, 133, 1526.

52 M. Barbatti, Wiley Interdisciplinary Reviews: Computational Molecular Science, 2011, 1, 620-633.

53 R. Crespo-Otero and M. Barbatti, Chemical Reviews, 2018, 118, 7026-7068.

54 G. Granucci and M. Persico, The Journal of Chemical Physics, 2007, 126, 134114.

55 J. E. Subotnik, W. Ouyang and B. R. Landry, The Journal of Chemical Physics, 2013, 139, 214107. 
56 C. Zhu, S. Nangia, A. W. Jasper and D. G. Truhlar, The Journal of Chemical Physics, 2004, 121, 7658-7670.

57 F. Furche and R. Ahlrichs, The Journal of Chemical Physics, 2002, 117, 7433-7447.

58 F. Furche and R. Ahlrichs, The Journal of Chemical Physics, 2004, 121, 12772-12773.

59 D. Heringer, T. A. Niehaus, M. Wanko and T. Frauenheim, Journal of Computational Chemistry, 2007, 28, 2589-2601.

60 D. Heringer, T. A. Niehaus, M. Wanko and T. Frauenheim, Journal of Computational Chemistry, 2011, 33, 593-593.

61 R. Mitrić, U. Werner and V. Bonačić-Koutecký, The Journal of Chemical Physics, 2008, 129, 164118.

62 U. Werner, R. Mitrić, T. Suzuki and V. Bonačić-Koutecký, Chemical Physics, 2008, 349, 319-324.

63 F. Plasser, G. Granucci, J. Pittner, M. Barbatti, M. Persico and H. Lischka, The Journal of Chemical Physics, 2012, 137, $22 \mathrm{~A} 514$.

64 J. Suchan, D. Hollas, B. F. E. Curchod and P. Slavíček, Faraday Discuss., 2018, 212, 307-330.

65 M. J. Frisch, G. W. Trucks, H. B. Schlegel, G. E. Scuseria, M. A. Robb, J. R. Cheeseman, G. Scalmani, V. Barone, B. Mennucci, G. A. Petersson, H. Nakatsuji, M. Caricato, X. Li, H. P. Hratchian, A. F. Izmaylov, J. Bloino, G. Zheng, J. L. Sonnenberg, M. Hada, M. Ehara, K. Toyota, R. Fukuda, J. Hasegawa, M. Ishida, T. Nakajima, Y. Honda, O. Kitao, H. Nakai, T. Vreven, J. A. Montgomery, Jr., J. E. Peralta, F. Ogliaro, M. Bearpark, J. J. Heyd, E. Brothers, K. N. Kudin, V. N. Staroverov, T. Keith, R. Kobayashi, J. Normand, K. Raghavachari, A. Rendell, J. C. Burant, S. S. Iyengar, J. Tomasi, M. Cossi, N. Rega, J. M. Millam, M. Klene, J. E. Knox, J. B. Cross, V. Bakken, C. Adamo, J. Jaramillo, R. Gomperts, R. E. Stratmann, O. Yazyev, A. J. Austin, R. Cammi, C. Pomelli, J. W. Ochterski, R. L. Martin, K. Morokuma, V. G. Zakrzewski, G. A. Voth, P. Salvador, J. J. Dannenberg, S. Dap- prich, A. D. Daniels, O. Farkas, J. B. Foresman, J. V. Ortiz, J. Cioslowski and D. J. Fox, Gaussian 09 (Revision D.01), Gaussian Inc., Wallingford, CT, 2009.

66 J. Frenzel, A. F. Oliveira, N. Jardillier, T. Heine and G. Seifert, Semi-relativistic, self-consistent charge Slater-Koster tables for density-functional based tight-binding (DFTB) for materials science simulations, TU Dresden, 2004-2009.

67 H. H. Heinze, A. Görling and N. Rösch, The Journal of Chemical Physics, 2000, 113, 2088-2099.

68 R. Mondal, C. Tönshoff, D. Khon, D. C. Neckers and H. F. Bettinger, Journal of the American Chemical Society, 2009, 131, 14281-14289.

69 C. Tönshoff and H. F. Bettinger, Angewandte Chemie International Edition, 2010, 49, 4125-4128.

70 M. Kasha, Discuss. Faraday Soc., 1950, 9, 14-19.

71 S. Knippenberg, J. H. Starcke, M. Wormit and A. Dreuw, Molecular Physics, 2010, 108, 2801-2813.

72 M. Chergui, Pure and Applied Chemistry, 2015, 87, 12. 525536.

73 B. Stevens, M. F. Thomaz and J. Jones, The Journal of Chemical Physics, 1967, 46, 405-406.

74 V. Lawetz, G. Orlandi and W. Siebrand, The Journal of Chemical Physics, 1972, 56, 4058-4072.

75 H. Morrison and A. Miller, Tetrahedron, 1981, 37, 3405-3409.

76 K. Schmidt, S. Brovelli, V. Coropceanu, D. Beljonne, J. Cornil, C. Bazzini, T. Caronna, R. Tubino, F. Meinardi, Z. Shuai and J.-L. Brédas, The Journal of Physical Chemistry A, 2007, 111, 10490-10499.

77 M. Rubio, M. Merchán, E. Ortí and B. O. Roos, Chemical Physics, 1994, 179, 395-409.

78 F. Bettanin, L. F. A. Ferrão, M. Pinheiro, A. J. A. Aquino, H. Lischka, F. B. C. Machado and D. Nachtigallova, Journal of Chemical Theory and Computation, 2017, 13, 4297-4306. 


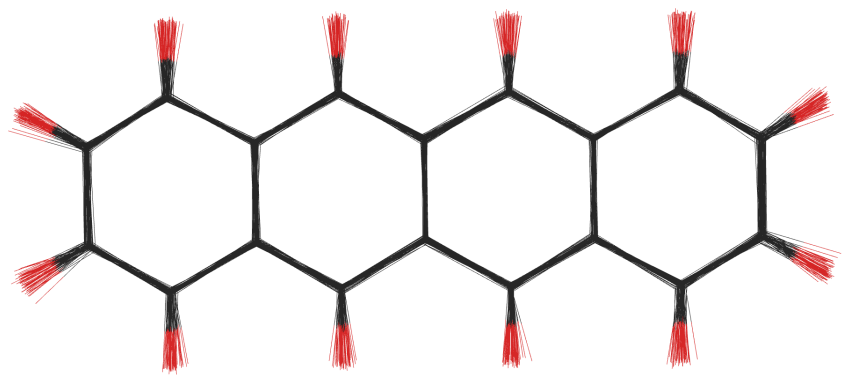

a) Top view

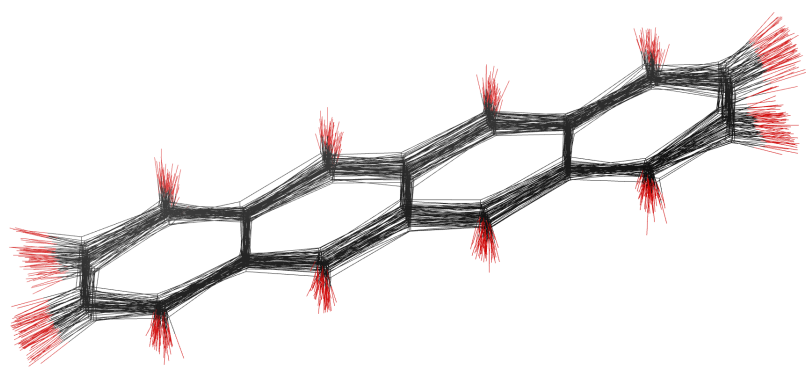

b) Side view

Fig. 163 initial configurations of tetracene sampled from the thermal distribution at $T=300 \mathrm{~K}$. Black color denotes carbon atoms, red color denotes hydrogen atoms.

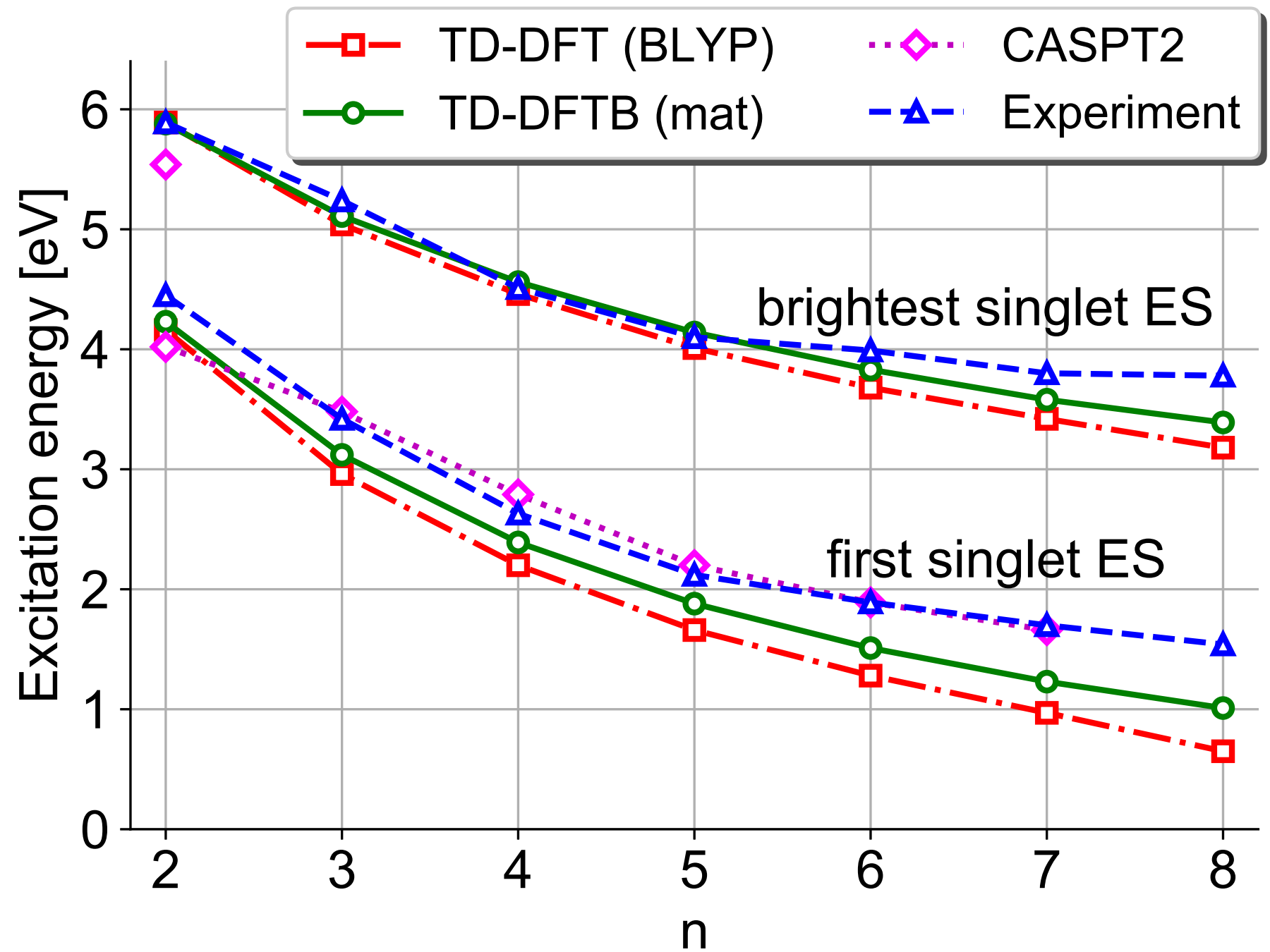

Fig. 2 Energies of the brightest and first singlet excited states (ES) in the theoretical CASPT2 ${ }^{77,78}$ (magenta diamonds, dotted line), TD-DFT (red squares, dash-dotted line), TD-DFTB (green circles, solid line) and experimental ${ }^{67-69}$ (blue triangles, dashed line) absorption spectra of polyacenes (n is the number of aromatic cycles in the molecule). The energy marks are connected by lines to guide the eye. All plotted values can be found in Tab. $\mathrm{S} 1$ (see ESI $\dagger$ ). 

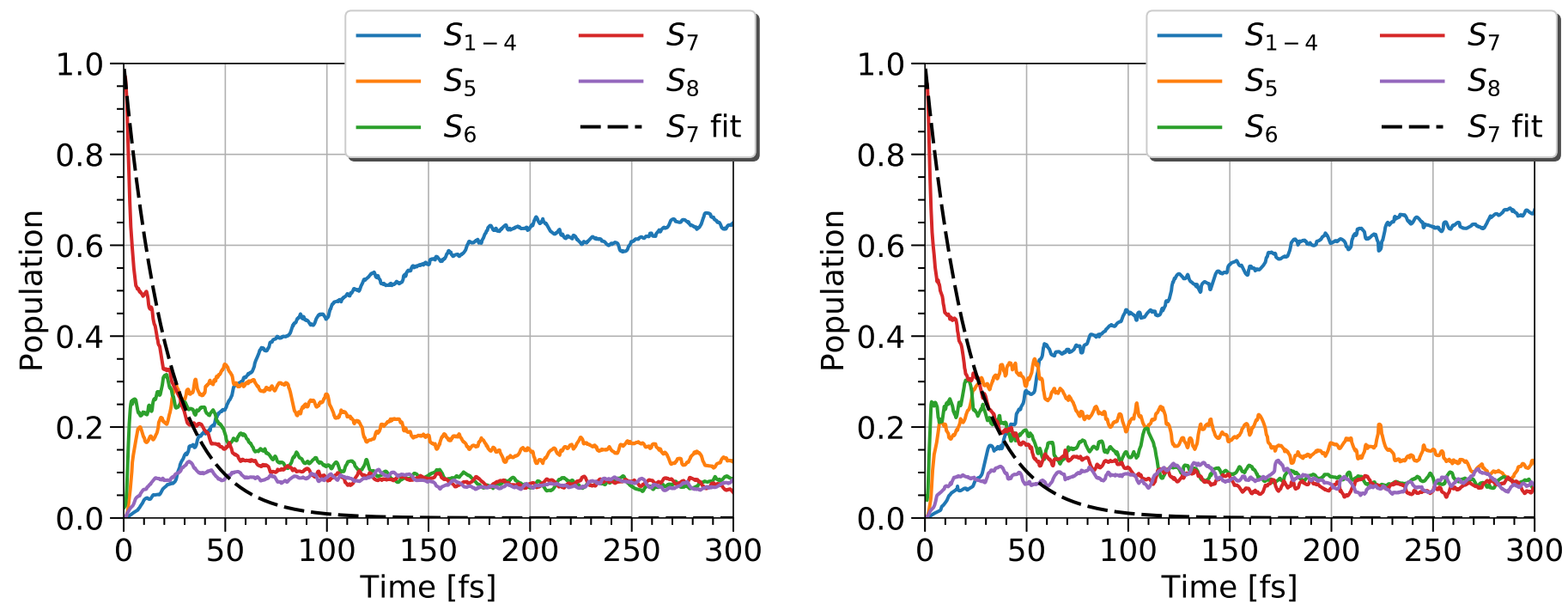

Fig. 3 Population analysis of the first 8 singlet excited states in anthracene for an ensemble of 127 (left panel) and 63 (right panel) trajectories. The initial state is $S_{7}$. The blue curves hereafter represent the sums of averaged populations in the lower-lying singlet states. For instance, the $S_{1-4}$ curve is $p_{1}+p_{2}+p_{3}+p_{4}$.

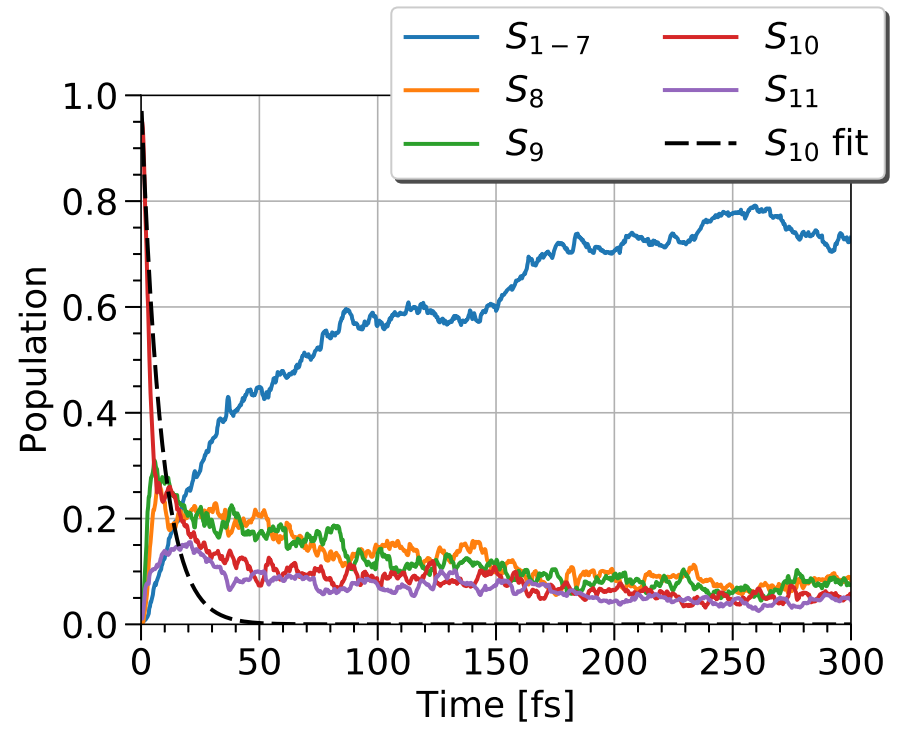

a) Pentacene $\left(S_{10}\right)$

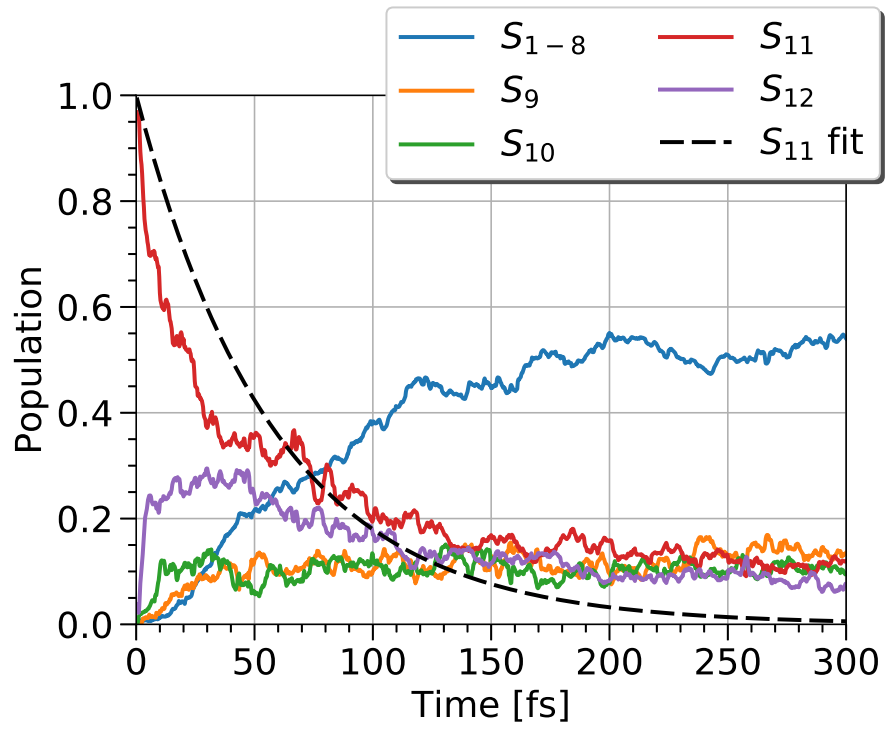

b) Heptacene $\left(S_{11}\right)$

Fig. 4 Population analysis of the first 11 and 12 singlet excited states in pentacene and heptacene, respectively, for an ensemble of 63 trajectories. The initial state is indicated between parentheses. 


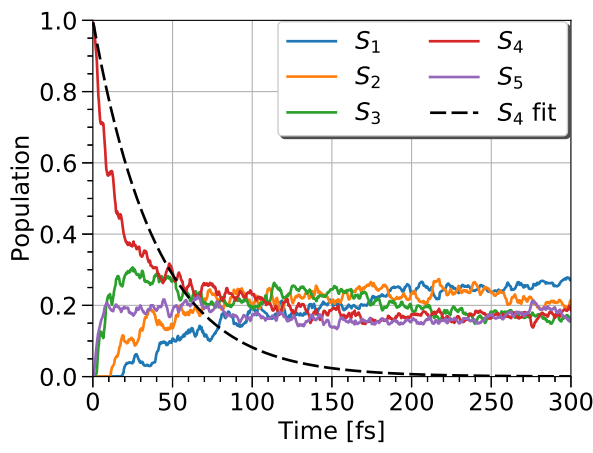

a) Naphthalene $\left(S_{4}\right)$

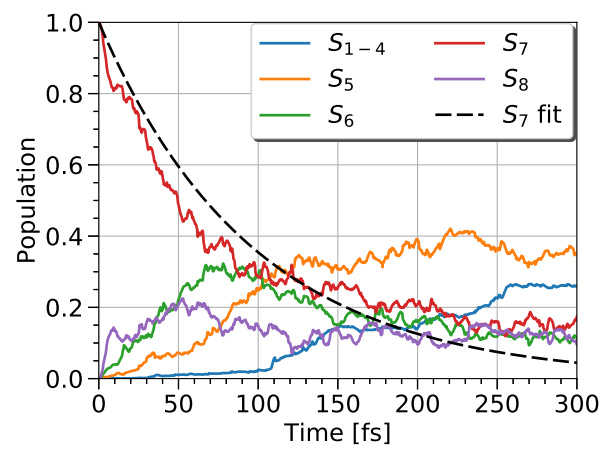

b) Tetracene $\left(S_{7}\right)$

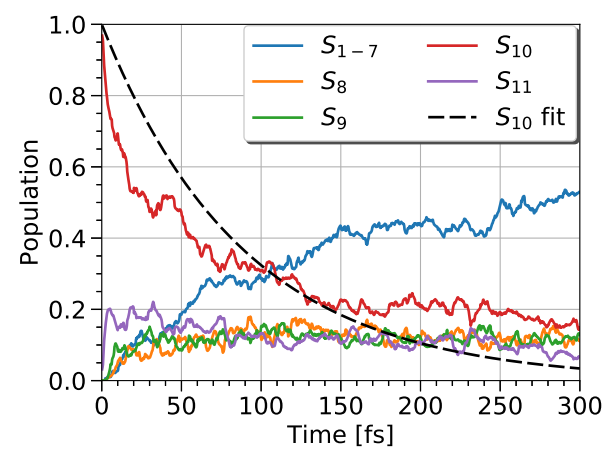

c) Hexacene $\left(S_{10}\right)$

Fig. 5 Population analysis of the first 5, 8 and 11 singlet excited states in naphthalene, tetracene and hexacene, respectively, for an ensemble of 127 trajectories (naphthalene) and 63 trajectories (tetracene, hexacene). The initial state is indicated between parentheses.

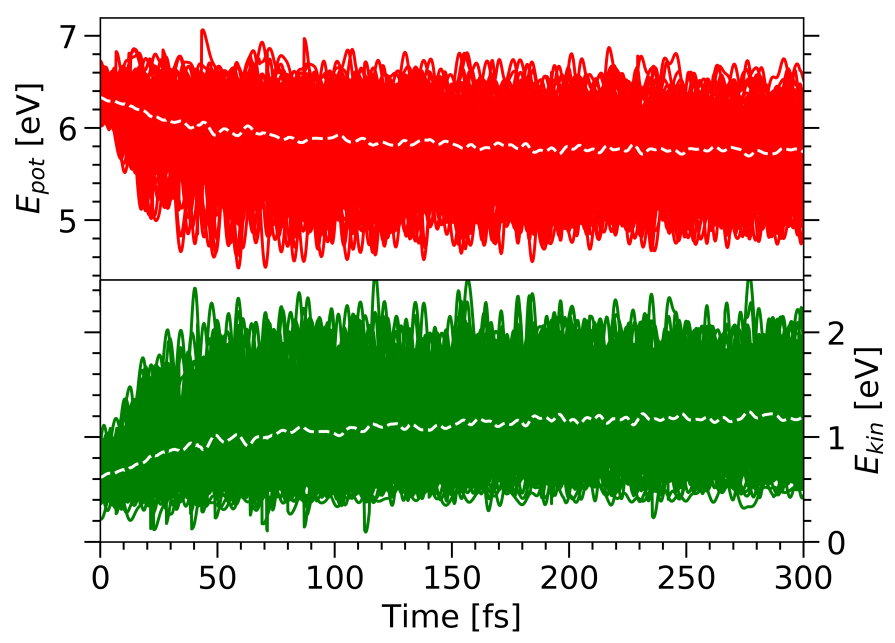

a) Naphthalene

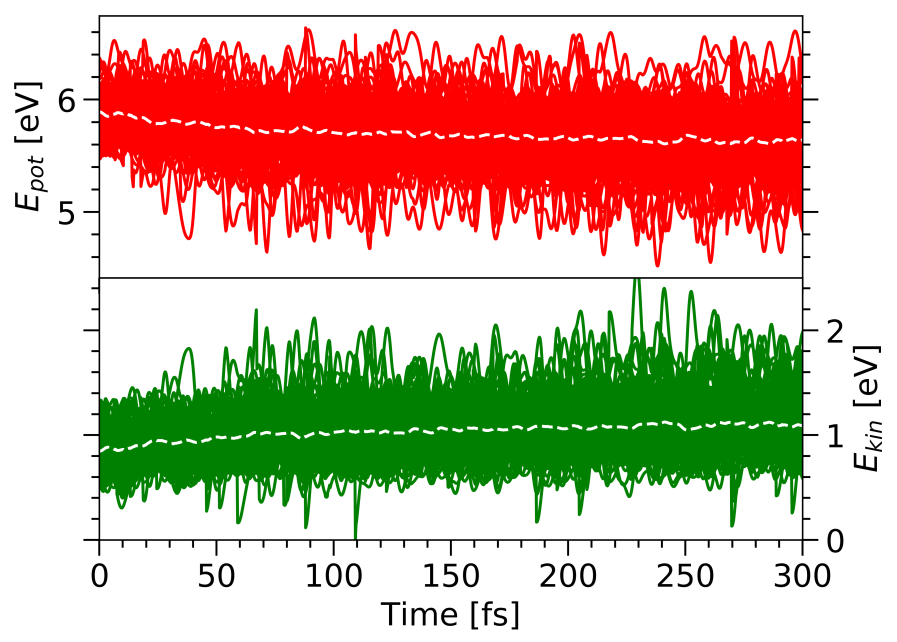

b) Anthracene

Fig. 6 Electronic potential (red color, upper panel) and total nuclear kinetic (green color, bottom panel) energy along a swarm of 127 trajectories in naphthalene and anthracene. The white dashed line is the average value for each panel. 


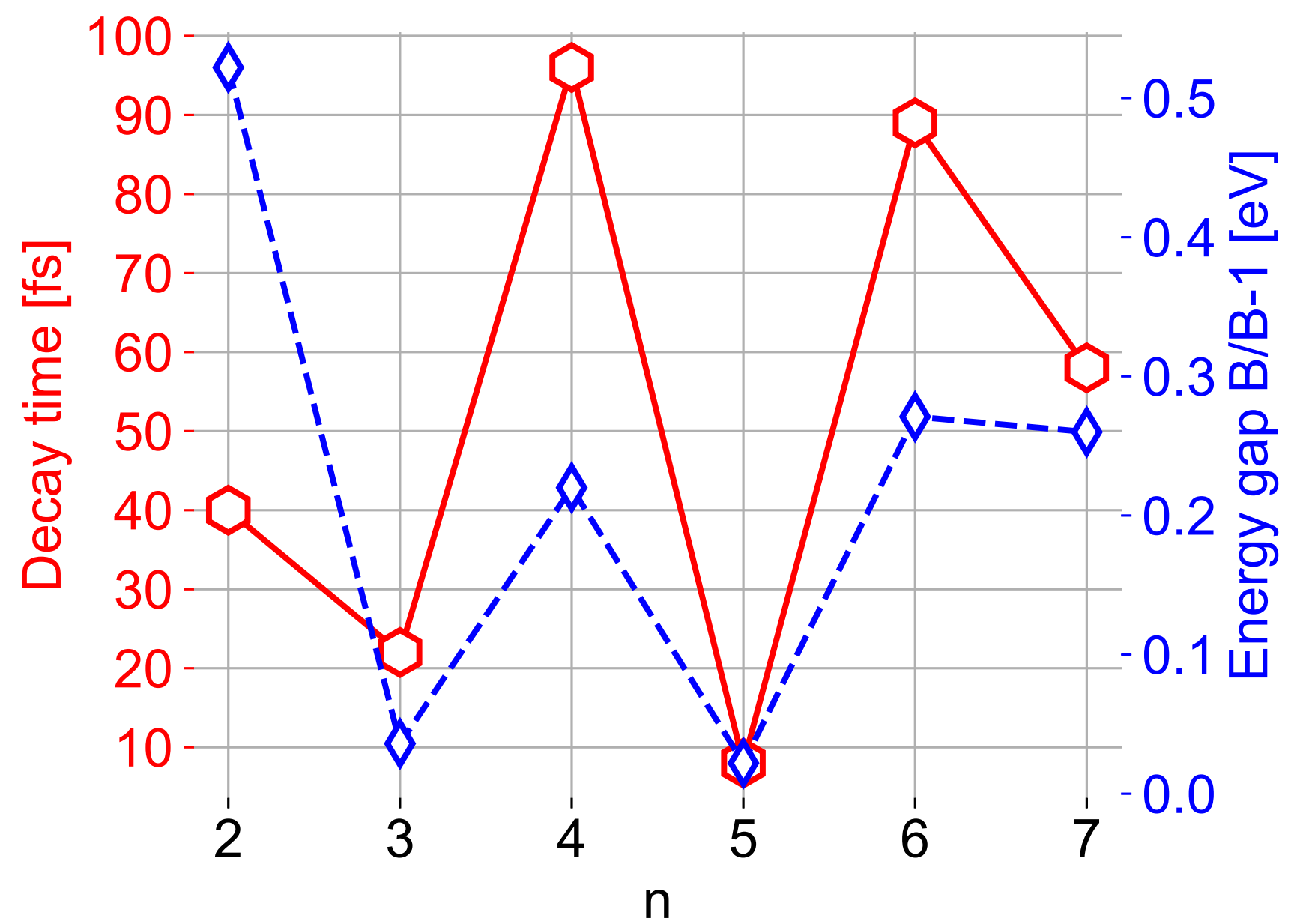

Fig. 7 Decay time of the brightest (B) singlet excited state (red hexagons) and the energy gap (blue diamonds) between the B and B-1 (the one with lower energy in TD-DFTB absorption spectra) states computed at the equilibrium geometry as a function of number of aromatic cycles in the polyacene. 

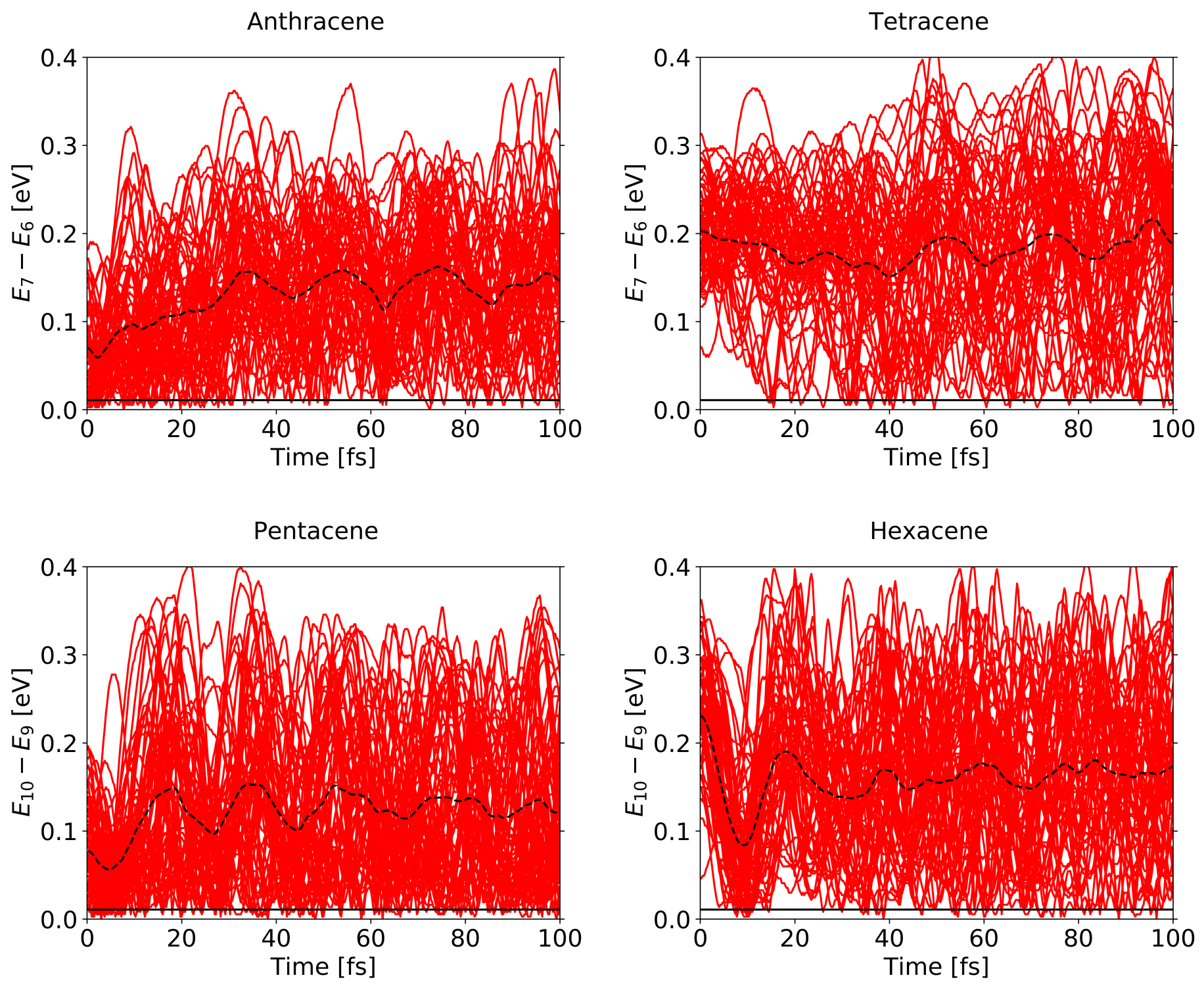

Fig. 8 Energy gap between the B and B-1 states plotted along each trajectory (red lines) and averaged over the ensemble of trajectories (black dashed line) for a set of polyacenes. The horizontal black line at $0.01 \mathrm{eV}$ is the Coln threshold. 